Pacific Journal of Mathematics

FINITELY GENERATED ALGEBRAS AND ALGEBRAS OF
SOLUTIONS TO PARTIAL DIFFERENTIAL EQUATIONS 


\title{
FINITELY GENERATED ALGEBRAS AND ALGEBRAS OF SOLUTIONS TO PARTIAL DIFFERENTIAL EQUATIONS
}

\author{
JOHN T. ANDERSON
}

\begin{abstract}
We consider two types of uniform algebras $A$ on the closure $\bar{\Omega}$ of a domain $\Omega \subset \mathbf{R}^{n}$ : those generated by finitely many smooth functions and those consisting of solutions to $L u=0$ where $L$ is a smooth complex vector field on $\Omega$. Under certain conditions we prove the existence of one of two types of analytic structure in the maximal ideal space $M_{A}$ of such an algebra: local foliations of $\Omega$ by complex manifolds on which the functions in the algebra are holomorphic, or foliations of a subset of $M_{A} \backslash \bar{\Omega}$ by analytic disks. Some open questions suggested by this line of inquiry are discussed.
\end{abstract}

1. Introduction. It is a central problem in the theory of uniform algebras to uncover general hypotheses on a proper subalgebra $A$ of $C(X)$ which imply the existence of analytic structure in the maximal ideal space $M_{A}$ of $A$ (see [Br] or [G]). The purpose of this paper is to exhibit under certain conditions such analytic structure in the maximal ideal spaces of the following two classes of algebras:

1. Finitely generated algebras. Suppose $f_{1}, \ldots, f_{k}$ are $C^{\infty}$ functions in a neighborhood of the closure of a bounded, smoothly bounded domain $\Omega \subset \mathbf{R}^{n}$, and suppose that $f_{1}, \ldots, f_{k}$ separate points on $\bar{\Omega}$. Let $\mathbf{C}\left[f_{1}, \ldots, f_{k}\right]$ be the algebra of polynomials in $f_{1}, \ldots, f_{k}$, and let $A$ be the closure of $\mathbf{C}\left[f_{1}, \ldots, f_{k}\right]$ in $C(\bar{\Omega})$. Then $A$ is a uniform algebra on $\bar{\Omega}$. Assuming that $A$ is nowhere locally dense in the continuous functions, and that $F=\left(f_{1}, \ldots, f_{k}\right)$ is an imbedding of $\bar{\Omega}$ into $\mathbf{C}^{k}$, then we obtain analytic structure in $M_{A}$ (Theorem 3.2).

To study the algebra of polynomials in $f_{1}, \ldots, f_{k}$, we consider the map $F=\left(f_{1}, \ldots, f_{k}\right)$ of $\bar{\Omega}$ into $\mathbf{C}^{k}$, which induces an isomorphism of $A$ with $P(K)$, the algebra of uniform limits of polynomials in $\left(z_{1}, \ldots, z_{k}\right)$ on $K=F(\bar{\Omega})$. If $F$ is a diffeomorphism, then we can use well-known results concerning real submanifolds of $C^{k}$ to study the algebra $P(K)$. These include a theorem of Hörmander and Wermer [H-W] on polynomial approximation on totally real submanifolds of $\mathbf{C}^{n}$, a theorem of Freeman on complex foliations of real submanifolds of $\mathbf{C}^{n}$, and a 
theorem of Hill and Taiani [H-T] on the existence of analytic disks with boundaries on prescribed submanifolds of $\mathbf{C}^{n}$.

2. Algebras of solutions to partial differential equations. Let $L$ be a first-order linear partial differential operator

$$
L=\sum_{j=1}^{n} a_{j}(x) \partial / \partial x_{j}
$$

with complex-valued coefficients $a_{j}$ defined and smooth in a neighborhood of $\bar{\Omega}, \Omega$ as in (1). Let $A_{0}=\left\{u \in C^{1}(\Omega): L u=0\right.$ in $\left.\Omega\right\}$. The closure $A$ of $A_{0}$ in $C(\bar{\Omega})$ will be a uniform algebra on $A$ provided the functions in $A$ separate points on $\Omega$. This is by no means guaranteed. (See $[\mathbf{N}]$, for example.) A connection between the class of finitely generated algebras and the algebra of functions annihilated by a vector field $L$ is provided by the Baouendi-Treves theorem, which gives conditions under which all solutions to $L u=0$ can be locally uniformly approximated by polynomials in a fixed set of solutions $f_{1}, \ldots, f_{k}$. Thus it is natural to assume that there exist smooth functions $f_{1}, \ldots, f_{k}$ in $A_{0}$ such that $F=\left(f_{1}, \ldots, f_{k}\right)$ is an imbedding of $\Omega$ into $\mathbf{C}^{k}$. We can then employ the same techniques used in the finitely generated case. However, we have been able to carry this out only for $n=3$ (Theorem 4.1). In dimension 4 , we are led to a solvability question which can be stated as follows: Let $L$ be a vector field on $\mathbf{C}^{2}$ of the form

$$
L=a_{1} \partial / \partial \bar{z}_{1}+a_{2} \partial / \partial \bar{z}_{2}
$$

with smooth coefficients $a_{1}, a_{2}$. Does the equation $L u=0$ always have non-holomorphic solutions?

Two examples will illustrate types of analytic structure possible in $M_{A}$ :

1. Let $\Omega$ be the unit ball in $\mathbf{R}^{3}$, with coordinates $\left(x_{1}, x_{2}, x_{3}\right)$, and consider the algebra generated by the functions $f_{1}(x)=x_{1}+i x_{2}$, $f_{2}(x)=x_{3}$. Then $A$ consists of continuous functions holomorphic in the variable $x_{1}+i x_{2}$. Thus $\Omega$ is foliated by sets $\left\{x_{3}=\right.$ constant $\}$ on which functions in the algebra are holomorphic. This algebra can also be defined as the closure in $C(\bar{\Omega})$ of $\left\{u \in C^{1}(\bar{\Omega}): L u=0\right\}$, where $L=\partial / \partial x_{1}+i \partial / \partial x_{2}$.

2. Let $\Omega$ be as in example 1 and let $A$ be the algebra generated by

$$
f_{1}(x)=x_{1}+i x_{2}, \quad f_{2}(x)=x_{3}+i\left(x_{1}^{2}+x_{2}^{2}\right) .
$$


The map $F=\left(f_{1}, f_{2}\right)$ imbeds $\bar{\Omega}$ onto a compact neighborhood $K$ of the origin in the hypersurface $\operatorname{Im}\left(z_{2}\right)=\left|z_{1}\right|^{2}$. The domain $\operatorname{Im}\left(z_{2}\right)>\left|z_{1}\right|^{2}$ is foliated by disks

$$
\Delta_{s, t}: z_{2}=s+i t, \quad\left|z_{1}\right|<t^{1 / 2}, \quad t>0
$$

whose boundaries lie on $M$. By the maximum principle, each element of $P(K)$ extends to the union of all those disks whose boundaries lie in $K$, and is holomorphic on each disk. Thus $M_{A}$ contains a four-dimensional set foliated by analytic disks to which elements in $A$ extend holomorphically. Locally this algebra can be defined as the closure of the solutions to $L u=0$ where $L$ is the Lewy operator: $L=\partial / \partial x_{1}+i \partial / \partial x_{2}-2 i\left(x_{1}+x_{2}\right) \partial / \partial x_{3}$.

In $\S 2$, we give definitions, preliminary lemmas and statements of the theorems needed in $\S \S 3$ and 4 . In $\S 3$, we give the theorem on analytic structure in the maximal ideal space of finitely generated algebras. In $\S 4$, we give a similar theorem for algebras of solutions to $L u=0$ in three dimensions, and discuss the solvability question posed above. We also discuss some questions related to the characterization of these algebras.

The results of this paper formed part of the author's doctoral dissertation written under the direction of John Wermer at Brown University. The author wishes to express his thanks to Professor Wermer for his direction and encouragement.

2. Notations and preliminaries. For any compact Hausdorff space $X, C(X)$ denotes the space of continuous complex-valued functions on $X$ provided with the topology arising from the uniform norm

$$
\|g\|_{X}=\sup _{x \in X}|g(x)| \text {. }
$$

A closed point-separating subalgebra $A$ of $C(X)$ containing the constants is called a uniform algebra on $X$. The maximal ideal space $M_{A}$ of $A$ can be identified with the set of non-zero homomorphisms $\varphi: A \rightarrow \mathbf{C}$, and is compact in the weak-* topology. $X$ is imbedded as a compact subset of $M_{A}$ by the map $x \rightarrow \varphi_{x}$, where $\varphi_{x}(f)=f(x)$ for $f \in A$. Each $f \in A$ extends to $\hat{f} \in C\left(M_{A}\right)$ by setting $\hat{f}(\varphi)=\varphi(f)$ for $\varphi \in M_{A}$.

If $K$ is any compact subset of $\mathbf{C}^{k}, P(K)$ will denote the closure in $C(K)$ of the algebra $\mathbf{C}\left[z_{1}, \ldots, z_{k}\right]$ of polynomials on $\mathbf{C}^{k} . P(K)$ is a uniform algebra on $K$. The polynomial hull $\hat{K}$ of $K$ is defined as

$$
\hat{K}=\left\{z \in \mathbf{C}^{k}:|P(z)| \leq\|P\|_{K} \text { for all } P \in \mathbf{C}\left[z_{1}, \ldots, z_{k}\right]\right\} .
$$


$K$ is said to be polynomially convex if $K=\hat{K} . M_{P(K)}$ can be identified with $\hat{K}$ via the map $\varphi \rightarrow\left(\varphi\left(z_{1}\right), \ldots, \varphi\left(z_{k}\right)\right)$.

We will be concerned with the case when $K$ is a compact subset of a regularly imbedded $C^{\infty}$ real submanifold $M$ of an open subset $U$ of $\mathbf{C}^{k}$. A good reference for what follows is the survey article of Wells [W]. For each $p \in M, H_{p} M$ denotes the space of vectors in the complexified tangent space $\mathrm{CT}_{p} M$ to $M$ at $p$ of the form

$$
Z=\sum_{j=1}^{n} a_{j} \partial / \partial z_{j}, \quad a_{j} \in \mathbf{C}, j=1, \ldots, n .
$$

Let $A_{p} M=\overline{H_{p} M}$, and let $h_{p} M$ be the dimension over $\mathbf{C}$ of $H_{p} M$.

We will assume that $M$ is given as follows: There exists a domain $\Omega \subset \mathbf{R}^{n}$, and complex-valued functions $f_{1}, \ldots, f_{k}$ such that the map $F(x)=\left(f_{1}(x), \ldots, f_{k}(x)\right)$ is a diffeomorphism of $\Omega$ with its image in $C^{k}$. The following facts are well-known; for the sake of completeness we include the proofs.

LEMMA 2.1. (1) $h_{p} M=n-r$, where $r$ is the rank over $\mathbf{C}$ of $\left\{d f_{1}(q), \ldots, d f_{k}(q)\right\}$ and $q=F^{-1}(p)$.

(2) $\left\{z \in M: h_{p} M \leq m\right\}$ is open (possibly empty) for each integer $m$.

(3) $n / 2 \geq h_{p} M \geq n-k$.

Proof. Since $F$ is a diffeomorphism, and $F^{*}\left(d z_{i}\right)(p)=d f_{i}(q), 1 \leq$ $i \leq k, r$ is also the rank over $\mathbf{C}$ of $\left\{d z_{i}(p), \ldots, d z_{k}(p)\right\}$. Since $A_{p} M$ is orthogonal to the $d z_{i}$ 's, $h_{p} M=\operatorname{dim}_{C} H_{p} M=n-r$. (2) and (3) are immediate consequences.

If $H_{p} M=\{0\}, M$ is said to be totally real at $p$; if this holds for all $p \in M, M$ is said to be totally real. If $h_{p} M$ is constant on $M$, $M$ is said to be a $C R$ submanifold of $U$, and we write $h M$ for $h_{p} M$. We will normally omit mention of $U$ and refer to $M$ simply as a $C R$ submanifold. $H M$ will denote the bundle whose fiber at $p$ is $H_{p} M$. Sections of $H M$ are known as holomorphic vector fields. If $h M=n-k$, $M$ is said to be generic.

If $M \subset \mathbf{C}^{k}$ and $M^{\prime} \subset \mathbf{C}^{k^{\prime}}$ are $C R$ submanifolds and $\varphi: M \rightarrow M^{\prime}$ is a diffeomorphism, then $\varphi$ is said to be a $C R$ equivalence of $M$ and $M^{\prime}$ if $\varphi_{*}\left(H_{p} M\right)=H_{\varphi(p)} M^{\prime}$ for all $p \in M$. If $\varphi$ is a $C R$ equivalence, then $f \in C R(M)$ if and only if $f \circ \varphi^{-1} \in C R\left(M^{\prime}\right)$. Any holomorphic map $\varphi: \mathbf{C}^{k} \rightarrow \mathbf{C}^{k^{\prime}}$ restricting to a diffeomorphism of $M$ with $M^{\prime}$ is a $C R$ equivalence of $M$ and $M^{\prime}$. The following lemma will allow us to replace a given $C R$ submanifold locally by an equivalent generic one. 
LEMMA 2.2. Let $M=F(\Omega), F=\left(f_{1}, \ldots, f_{k}\right)$ as above be a $C R$ submanifold with $h M=j$. Then in a neighborhood $V$ of a given point $q \in M$, we can find a linear map $\Psi: \mathbf{C}^{k} \rightarrow \mathbf{C}^{n-j}$ so that $\Psi$ is a $C R$ equivalence of $V$ with a generic $C R$ submanifold $N \subset \mathbf{C}^{n-j}$.

Proof. Let $p=F^{-1}(q)$. By Lemma 2.1, $\left\{d f_{1}(p), \ldots, d f_{k}(p)\right\}$ has rank $r=n-j$, so we can choose $g_{1}, \ldots, g_{n-j}$ linear combinations of the $f_{i}$ so that

if $n-j$ is odd, or if $n-j$ is even,

$$
\begin{gathered}
d g_{1}(p)=d x_{1}+i d x_{2}, \\
\vdots \\
d g_{n-j}(p)=d x_{n-j}+i d x_{n-j+1}
\end{gathered}
$$

$$
\begin{gathered}
d g_{1}(p)=d x_{1}+i d x_{2} \\
\vdots \\
d g_{n-j-1}(p)=d g_{n-j-1}+i d x_{n-j}, \\
d g_{n-j}(p)=d x_{n-j+1} .
\end{gathered}
$$

In either case, the rank of

$$
d\left(\operatorname{Re} g_{1}\right), \ldots, d\left(\operatorname{Re} g_{n-j}\right), d\left(\operatorname{Im} g_{1}\right), \ldots, d\left(\operatorname{Im} g_{n-j+1}\right)
$$

is at least $2(n-j) \geq n$ by Lemma 2.1 . So locally $G=\left(g_{1}, \ldots, g_{n-j}\right)$ is a diffeomorphism. We define $\Psi$ so that $G=\Psi \circ F$, i.e., if $g_{i}=\sum_{l=1}^{k} a_{i l} f_{l}$ then $\Psi=\left(\psi_{1}, \ldots, \psi_{n-j}\right)$ where $\psi_{i}(z)=\sum_{l-1}^{k} a_{i l} z_{l}$ is the desired map.

A function $u \in C(M)$ is said to be a $C R$ function on the open subset $V$ of $M$ if $\bar{Z} u=0$ (in the sense of distributions) whenever $Z$ is a holomorphic vector field on $M$, and we write $u \in C R(V)$. If $u$ is a uniform limit of smooth functions $u_{n}$ such that $L u_{n}=0$, then $L u=0$ in the sense of distributions. Thus, if $K$ is a compact subset of $M$, and $u \in P(K)$, then $u$ is a $C R$ function on the interior of $K$. The following theorem of Baouendi and Treves provides a local converse (Corollary 2.4 below) to this statement and is fundamental in the study of $C R$ functions.

THEOREM 2.3 [B-T]. Let $L_{1}, \ldots, L_{m}$ be smooth complex vector fields on a domain $\Omega \subset \mathbf{R}^{n}$, satisfying the following conditions:

(1) $\left\{L_{1}(p), \ldots, L_{m}(p)\right\}$ has rank $m$ over $\mathbf{C}$ at each $p \in \Omega$.

(2) For each $i, j,\left[L_{i}, L_{j}\right]$ is a linear combination of $L_{1}, \ldots, L_{m}$.

(3) For some $p_{0} \in \Omega$ there exist functions $f_{1}, \ldots, f_{n-m} \in C^{\infty}(\Omega)$ such that $d f_{1}\left(p_{0}\right), \ldots, d f_{n-m}\left(p_{0}\right)$ are linearly independent over $\mathbf{C}$ and $L_{i} f_{j}=0$ in $\Omega, 1 \leq i \leq m ; 1 \leq j \leq n-m$. 
Then there exists a neighborhood $V$ of $p_{0}$ such that if $u$ is a continuous function satisfying $L_{i} u=0$ in the sense of distributions in $\Omega, 1 \leq i \leq m$, then $u$ is a uniform limit on $V$ of polynomials in $f_{1}, \ldots, f_{n-m}$.

Corollary 2.4. Let $M$ be a $C R$ submanifold, $M=F(\Omega) \subset \mathbf{C}^{k}$, and suppose $u \in C R(M)$. For each $p \in M$, there exists a compact neighborhood $K$ of $p$ in $M$ such that $u \in P(K)$.

Proof. Let $Z_{1}, \ldots, Z_{m}$ be a basis for the sections of $H M$ near $p$. Then $d z_{1}(p), \ldots, d z_{k}(p)$ has rank $n-m$ by Lemma 2.1 , so we can apply Theorem 1.3 to $F_{*}^{-1}\left(\bar{Z}_{i}\right)=L_{i}$ (note that $\left[\bar{Z}_{i}, \bar{Z}_{j}\right] \in H_{p} M$, so condition (2) is satisfied) and $n-m$ of the $f_{i}$ to obtain the result.

We will be interested in local properties of $\mathbf{C}\left[z_{1}, \ldots, z_{k}\right]$ on $M$; i.e., $P(K)$ when $K$ is a neighborhood of a given point $p \in M$. In the totally real case we have the following result of Hörmander and Wermer:

THEOREM 2.5. If $M$ is totally real, and $K$ is a polynomially convex subset of $M$, then $P(K)=C(K)$.

Combining Theorem 2.5 with the following lemma gives us the desired local information.

LEMMA 2.6. If $M$ is totally real, then $M$ has a neighborhood basis of polynomially convex sets.

Proof. The proof of this lemma is essentially contained in [H-W]. It can also be derived from a reading of the proof of Theorem 2.3 in the case where there are no vector fields $(m=0)$. For this observation we are indebted to J. Polking. See [A] for details.

If $M$ is not totally real, then the question of whether or not the sections of $H M$ are tangent to complex submanifolds of $M$ becomes important. A collection $\Sigma$ of submanifolds of an open subset $U$ of $M$ is called a foliation of $U$ by leaves of dimension $m(m<n)$ if we can choose local coordinates $\left(x_{1}, \ldots, x_{n}\right)$ in $U$ such that $\Sigma$ is the collection of submanifolds $\Sigma_{c}=\left\{x_{1}=c_{1}, \ldots, x_{n-m}=c_{n-m}\right\},\left(c_{1}, \ldots, c_{n-m}\right) \in$ $\mathbf{R}^{n-m}$. Let $V$ be a neighborhood of $p \in \mathbf{C}^{k}$. A complex foliation $\Sigma$ of $V \cap M$ is a foliation of $V \cap M$ such that each leaf of $\Sigma$ is a complex submanifold of $V$. 
Given $p \in M$, there exists a neighborhood $V$ of $p$ in $\mathbf{C}^{k}$ and $2 k-n$ real valued functions $\rho_{1}, \ldots, \rho_{2 n-k} \in C^{\infty}(V)$ such that

$$
M \cap V=\left\{z \in V: \rho_{1}(z)=0, \ldots, \rho_{2 n-k}(z)=0\right\}
$$

and $d \rho_{1}, \ldots, d \rho_{2 n-k}$ are linearly independent on $M \cap V$. A $C R$ submanifold $M$ which is not totally real is said to be Levi-flat at $p \in M$ if whenever

$$
\sum_{j=1}^{n} a_{j} \partial / \partial \bar{z}_{j} \in A_{p} M
$$

then

$$
\sum_{i, j=1}^{n} a_{i} \bar{a}_{j} \partial^{2} \rho_{s}(p) / \partial z_{i} \partial \bar{z}_{j}=0, \quad s=1, \ldots, 2 k-n .
$$

This condition is independent of the choice of defining functions $\rho_{1}, \ldots, \rho_{2 n-k}$, and is invariant under $C R$ equivalences. A theorem of Freeman states that Levi-flatness implies the existence of local complex foliations.

THEOREM 2.7 [F]. If the CR submanifold $M$ is Levi-flat for all $p$ in a neighborhood of $p_{0}$ in $M$, then there exists a neighborhood $V$ of $p_{0}$ in $\mathbf{C}^{k}$ and a complex foliation $\Sigma$ of $V \cap M$ by leaves of dimension $h M$, such that for any leaf $\Sigma_{c}$, and any point $q \in \Sigma_{c}, H_{q} M=H_{q} \Sigma_{c}$.

Clearly, if $K$ is a compact neighborhood of $p_{0}$, any $f \in P(K)$ is holomorphic on each leaf of $\Sigma$.

Finally, suppose $M$ is not Levi-flat at $p_{0}$. One can show the existence of analytic structure in $\hat{K} \backslash K$ for compact neighborhoods $K$ of $p_{0}$ by a method due to Bishop [B], and developed by Hill and Taiani. An analytic disk $D$ is the image of a continuous map $G$ from the closed unit disk $\{|z| \leq 1\} \subset \mathbf{C}$ into $\mathbf{C}^{k}$ which is holomorphic on $|z|<1$. By the maximum principle, if $\partial D \subset K$, then $D \subset \hat{K}$.

THEOREM 2.8 [H-T]. Assume $M$ is generic and $M$ is not Levi-flat at $p_{0}$. Then there exists a neighborhood $V$ of $p_{0}$ in $M$ and an $(n+1)$ dimensional submanifold $M^{\prime}$ foliated by analytic disks such that $V$ is the union of the boundaries of the disks.

3. Finitely generated algebras. Throughout this section $\Omega$ will be a domain in $\mathbf{R}^{n}$ with compact closure $\bar{\Omega}$ and $C^{\infty}$ boundary. We will consider algebras of the form $\mathbf{C}\left[f_{1}, \ldots, f_{k}\right]$ where $f_{1}, \ldots, f_{k}$ are smooth 
complex-valued functions on $\Omega$. In particular we assume:

1. The map $F(x)=\left(f_{1}(x), \ldots, f_{k}(x)\right)$ is a diffeomorphism of a neighborhood $U$ of $\bar{\Omega}$ with its image in $\mathbf{C}^{k}$.

2. $\mathbf{C}\left[f_{1}, \ldots, f_{k}\right]$ is nowhere locally dense: for each open subset $V$ of $\bar{\Omega}, \mathbf{C}\left[f_{1}, \ldots, f_{k}\right]$ is not dense in $C(\bar{V})$.

We note that under these assumptions $F$ induces an isomorphism of the closure of $\mathbf{C}\left[f_{1}, \ldots, f_{k}\right]$ in $C(V)$ with $P(F(V))$ for any compact $V \subset \Omega$. We let $A$ be the closure of $\mathbf{C}\left[f_{1}, \ldots, f_{k}\right]$ in $C(\bar{\Omega})$.

LEMMA 3.1. Under assumptions (1) and (2), for each $p \in \Omega$, the rank of $\left\{d f_{1}(p), \ldots, d f_{k}(p)\right\}$ is less than $n$.

Proof. If the rank of $\left\{d f_{1}(p), \ldots, d f_{k}(p)\right\}=n$, and $M=F(\Omega)$, then $M$ is totally real in a neighborhood $U$ of $F(p)$, by Lemma 2.1. By Lemma 2.6 there exists a compact neighborhood $K$ of $F(p)$ with $K=\hat{K}$ and $K \subset U$. By Theorem 2.5, $P(K)=C(K)$. But then $\mathbf{C}\left[f_{1}, \ldots, f_{k}\right]$ is dense in $F^{-1}(K)$, contradicting the assumption that $\mathrm{C}\left[f_{1}, \ldots, f_{k}\right]$ is nowhere locally dense.

THEOREM 3.2. Under assumptions (1) and (2) one of the following alternatives holds:

(a) For each point $p$ in an open dense subset of $\Omega$, there is a neighborhood $V$ of $p$ and a complex foliation $\Sigma$ of $V$ such that each $f \in A$ is holomorphic on the leaves of $\Sigma$, or

(b) There exists an $(n+1)$-dimensional submanifold $N$ of some $\mathbf{C}^{N}$ foliated by analytic disks and a $1-1$ continuous map $\phi: N \rightarrow M_{A}$ such that for each $f \in A, \hat{f} \circ \phi$ is holomorphic on each disk.

Proof. Let $M=F(U)$. For $j=0,1,2, \ldots$, let $\Omega_{j}=\{x \in \Omega$ : $\left.h_{F(x)} M=j\right\}$. By Lemma 3.1, $\Omega_{0}$ is empty. Note that $\bigcup_{j=1}^{n} \dot{\Omega}_{j}$ is open and dense in $\Omega$, and that $F\left(\dot{\Omega}_{j}\right)$ is a $C R$ submanifold.

Let $X_{j}=\left\{x \in \dot{\Omega}_{j}: M_{j}\right.$ is not Levi-flat at $\left.F(x)\right\}$. We have two alternatives:

1. $X_{j}$ is empty for all $j$.

2. For some $j, X_{j}$ is not empty.

If (1) holds, then for each $p \in \dot{\Omega}_{j}$, by Theorem 2.7 , there exists complex foliation $\Sigma$ of a neighborhood $V$ of $F(p)$ by complex manifolds of dimension $j$. By the remark following Theorem 2.7, alternative (a) then holds.

If (2) holds, fix $x_{0}$ in $\dot{\Omega}_{j}$ such that $M$ is not Levi-flat at $F\left(x_{0}\right)$. By Lemma 2.2, we can find a neighborhood $U$ of $z_{0}=F\left(x_{0}\right)$ in $M_{j}$ and 
a linear $C R$ equivalence $\Psi: U \rightarrow M^{\prime} \subset \mathbf{C}^{n-j}$ so that $M^{\prime}$ is generic at $\Psi\left(z_{0}\right)=w_{0}$ and not Levi-flat at $w_{0}$. Now for each $f \in A, f \circ F^{-1} \in$ $P(U) \subset C R(U)$. Since $\Psi$ is a $C R$ equivalence, $f \circ F^{-1} \circ \Psi^{-1} \in C R\left(M^{\prime}\right)$. By the Baouendi-Treves theorem there exists a compact neighborhood $K$ of $w_{0}$ in $M^{\prime}$ so that $f \circ F^{-1} \circ \Psi^{-1} \in P(K)$. Let $N$ be the $(n+1)$ dimensional submanifold given by the Hill-Taiani Theorem, which is foliated by analytic disks whose boundaries fill out $K$. By the remarks preceding Theorem 2.8 , for each $f \in A, f \circ F^{-1} \circ \Psi^{-1}$ extends to an element $\tilde{f}$ of $P(N)$. Consider the map $\phi: N \rightarrow M_{A}$ given by $\phi(w)=$ $\varphi_{w}$, where $\varphi_{w}(f)=\tilde{f}(w)$. If $G=\Psi \circ F, G=\left(g_{1}, \ldots, g_{n-j}\right)$, then $g_{i} \in A$ and $\tilde{g}_{i}(w)=w_{i}$, where $\left(w_{1}, \ldots, w_{n-j}\right)$ are coordinates on $\mathbf{C}^{n-j}$. Thus $\phi(w)=w_{i}$, which implies that $\phi$ is one-to-one. If $h \in A$ and $w \in N$ are fixed then the continuity of $\tilde{h}$ implies that for each $\varepsilon>0$,

$$
\left\{w^{\prime} \in N:\left|\phi(w)(h)-\phi\left(w^{\prime}\right)(h)\right|<\varepsilon\right\}
$$

is open in $N$, so $\phi$ is continuous.

4. Algebras of solutions to partial differential equations. Let $\Omega$ be a domain in $\mathbf{R}^{n}$ as in $\S 3$. $L$ will be a vector field on $\Omega$,

$$
L=\sum_{j=1}^{n} a_{j}(x) \partial / \partial x_{j}
$$

where the coefficients $a_{j}$ are complex valued functions of class $C^{\infty}$ in a neighborhood of $\bar{\Omega}$. Set $A_{0}=\left\{u \in C^{1}(\bar{\Omega}): L u=0\right.$ in $\left.\Omega\right\}$. We note that $A_{0}$ is closed under addition and multiplication. Let $A$ denote the closure of $A_{0}$ in $C(\bar{\Omega})$. If $u \in A$, then $L u=0$ in $\Omega$ in the sense of distributions, so $A \subset A_{d}$, where $A_{d}=\{u \in C(\bar{\Omega}): L u=$ 0 in the sense of distributions on $\Omega\} . A_{d}$ is a closed linear subspace of $C(\bar{\Omega})$. This presents some interesting questions on the characterization of $A$ :

1. Is $A_{d}$ closed under multiplication?

2. Is $A=A_{d}$ ?

Note that the first question is local, for if a distribution vanishes on a neighborhood of each point in $\Omega$, it vanishes everywhere on $\Omega$. A positive answer to the second question would of course imply a positive answer to the first.

Suppose that $L$ satisfies the Baouendi-Treves conditions, which in the case of a single operator reduce to:

1. $L$ is non-vanishing on $\Omega$. 
2. For each $p_{0} \in \Omega$, there exist functions $f_{1}, \ldots, f_{n-1} \in C^{\infty}(\Omega)$ such that $d f_{1}\left(p_{0}\right), \ldots, d f_{n-1}\left(p_{0}\right)$ are linearly independent over $\mathbf{C}$ and $L f_{i}=0$ on $\Omega, 1 \leq i \leq n-1$.

Then, by the Baouendi-Treves theorem, any element of $A_{d}$ is locally a uniform limit of polynomials in $f_{1}, \ldots, f_{n-1}$. The same holds for products of elements in $A_{d}$, which implies that $A_{d}$ is closed under multiplication. We do not know whether under these conditions $A=$ $A_{d}$.

If $L$ is hypoelliptic, for instance if $L$ is the Cauchy-Riemann operator on $\Omega \subset \mathrm{C}$, then $A=A_{d}$. Moreover, it is not hard to show that if $\Omega$ is foliated by two-dimensional manifolds carrying a complex structure with respect to which $L$ is the Cauchy-Riemann operator, then $A=A_{d}$. Also if $L$ is equivalent to the tangential $C R$ operator on an open subset of the boundary of the unit sphere in $\mathbf{C}^{2}$, then an argument of $\mathrm{H}$. Lewy shows that $A=A_{d}$. Both these cases are treated in detail in [A].

Next we turn to the question of analytic structure in $M_{A}$. We will assume the following:

1. There exist functions $f_{1}, \ldots, f_{k} \in C^{\infty}(\bar{\Omega}) \cap A_{0}$ such that the map $F=\left(f_{1}, \ldots, f_{k}\right)$ is a diffeomorphism of $\bar{\Omega}$ with a submanifold $M$ of an open subset of $\mathbf{C}^{k}$.

THEOREM 4.1. Under condition 1 , if $n=3$, then either

(a) For each $p \in \Omega$, there exists a neighborhood $U$ of $p$ and a complex foliation of $U$ such that each $f \in A$ is holomorphic on the leaves of the foliation, or

(b) There exists an open subset $V$ of $\mathbf{C}^{2}$ foliated by analytic disks and a one-to-one continuous map $\phi: V \rightarrow M_{A}$ such that whenever $f \in A$, $\hat{f} \circ \phi$ is holomorphic on each disk.

Proof. Let $M=F(\Omega) \subset \mathbf{C}^{k}$. Then $Z=\overline{F_{*}(L)}$ is a non-vanishing holomorphic vector field on $M$. Since $M$ is three-dimensional, $M$ is a $C R$ submanifold with $h M=1$. We have two cases:

1. Suppose $M$ is Levi-flat everywhere. By Theorem 2.7, for each $q \in M$, there exists a neighborhood $W$ of $q$ in $M$ and a complex foliation of $W$ such that $F_{*}(L)$ is the Cauchy-Riemann operator on the leaves of the foliation; hence $f \circ F^{-1}$ is holomorphic on the leaves of the foliation for each $f \in A$. Thus alternative (a) holds.

2. Suppose there exists $p \in \Omega$ such that $M$ is not Levi-flat at $q=$ $F(p)$. By Lemma 2.2, there exists a linear map $\Psi: \mathbf{C}^{k} \rightarrow \mathbf{C}^{2}$ so that 
$M^{\prime}=\Psi(M)$ is a generic $C R$ submanifold in $\mathbf{C}^{2}$, not Levi-flat at $w=$ $\Psi(q)$. Moreover, if $G=\Psi \circ F, G=\left(g_{1}, g_{2}\right)$, then $d g_{1}(p)$ and $d g_{2}(p)$ are linearly independent over $\mathbf{C}$, so the requirements of the BaouendiTreves theorem are met. Thus in a neighborhood of $p$ in $\Omega$, each $f \in A$ is a uniform limit of polynomials in $g_{1}$ and $g_{2}$. It follows that $f \circ G^{-1} \in P(K)$ for some compact neighborhood $K$ of $w$ in $M^{\prime}$ whenever $f \in A$. By the Hill-Taiani theorem there exists a fourdimensional submanifold $V$ (thus an open subset) of $\mathbf{C}^{2}$ foliated by analytic disks with boundaries on $M^{\prime}$ filling out a neighborhood $K^{\prime} \subset$ $K$ of $w$ in $M^{\prime}$. We obtain a map $\phi: V \rightarrow M_{A}$ given by

$$
\phi(w)(f)=\tilde{f}(w)
$$

where $\tilde{f}$ is the extension of $f \circ G^{-1}$ to $V$. We conclude exactly as in the proof of Theorem 3.2 that $\phi$ is $1-1$ and continuous.

The proof of Theorem 4.1 shows that the existence of an imbedding $F$ with components in $A$ implies that the algebra is locally finitely generated, by the Baouendi-Treves theorem, and so we expect the situation to be similar to that in Theorem 3.2. However, when we consider the case $n=4$, the existence of such an imbedding does not guarantee enough solutions (three with linearly independent differentials are required) to apply the Baouendi-Treves theorem. For example, if $L=a \partial / \partial \bar{z}_{1}+b \partial / \partial \bar{z}_{2}$ where $a$ and $b$ are smooth functions on a domain $\Omega$ in $\mathbf{C}^{2}$, then $Z=\left(z_{1}, z_{2}\right)$ is an imbedding of $\Omega$ into $\mathbf{C}^{2}$ whose components are annihilated by $L$. For such an $L$, there need not exist three linearly independent solutions to $L u=0$. This leads to the following question:

If $L=a \partial / \partial \bar{z}_{1}+b \partial / \partial \bar{z}_{2}$ must there be non-holomorphic solutions to $L u=0$ ?

If $a$ and $b$ are real-analytic, then the answer is yes, by a simple application of the Cauchy-Kowalewski Theorem. Other conditions under which the answer is yes are given in [A], but the general question remains open.

\section{REFERENCES}

[A] J. Anderson, Algebras of Solutions to Partial Differential Equations, Ph.D thesis, Brown University, 1986.

[B] E. Bishop, Differentiable manifolds in complex Euclidean space, Duke Math. J., 32 (1965), 1-21.

[Br] A. Browder, Introduction to Function Algebras, W. A. Benjamin, 1969. 
[B-T] M. S. Baouendi and F. Treves, $A$ property of the functions and distributions annihilated by a locally integrable system of complex vector fields, Annals of Math., 113 (1981), 387-421.

[F] M. Freeman, Local complex foliations of real submanifolds, Math. Ann., 209 (1974), 1-30.

[G] T. Gamelin, Uniform Algebras, Prentice Hall, 1969.

[H-T] C. D. Hill and G. Taiani, Families of analytic disks with boundaries on a prescribed CR submanifold, in Raccolta Degli Scritti Dedicata a Hans Lewy, Scuola Normale Superiore di Pisa, 1978.

[H-W] L. Hörmander and J. Wermer, Uniform approximation on compact subsets in C $^{n}$, Math. Scand., 23 (1968).

[N] L. Nirenberg, Lectures on linear partial differential equations, Conference Board of the Mathematical Sciences, Regional Conference Series in Mathematics, American Mathematical Society, 1973.

[W] R. O. Wells, Function theory on differentiable submanifolds, from Contributions to Analysis, Papers Dedicated to Lipman Bers, Academic Press, 1974.

Received June 1, 1987.

College of the Holy Cross

WORCESTER, MA 01610 


\section{PACIFIC JOURNAL OF MATHEMATICS}

\section{EDITORS}

V. S. VARADARAJAN

(Managing Editor)

University of California

Los Angeles, CA 90024

Herbert Clemens

University of Utah

Salt Lake City, UT 84112

R. FINN

Stanford University

Stanford, CA 94305
HERMANN FLASCHKA

University of Arizona

Tucson, AZ 85721

RAMESh A. GANGOLLI

University of Washington Seattle, WA 98195

VAUGHAN F. R. JONES

University of California

Berkeley, CA 94720
ROBION KIRBY

University of California

Berkeley, CA 94720

C. C. MOORE

University of California

Berkeley, CA 94720

HAROLD STARK

University of California, San Diego

La Jolla, CA 92093

\section{ASSOCIATE EDITORS}
R. AREnS
E. F. BECKENBACH
B. H. NEUMANN
F. WOLF
K. YOSHIDA (1906-1982)

\section{SUPPORTING INSTITUTIONS}

UNIVERSITY OF ARIZONA

UNIVERSITY OF OREGON

UNIVERSITY OF BRITISH COLUMBIA UNIVERSITY OF SOUTHERN CALIFORNIA

CALIFORNIA INSTITUTE OF TECHNOLOGY

UNIVERSITY OF CALIFORNIA

STANFORD UNIVERSITY

MONTANA STATE UNIVERSITY

UNIVERSITY OF HAWAII

UNIVERSITY OF NEVADA, RENO

UNIVERSITY OF TOKYO

NEW MEXICO STATE UNIVERSITY

UNIVERSITY OF UTAH

OREGON STATE UNIVERSITY

WASHINGTON STATE UNIVERSITY

UNIVERSITY OF WASHINGTON 


\section{Pacific Journal of Mathematics}

\section{Vol. 133, No. $1 \quad$ March, 1988}

John Anderson, Finitely generated algebras and algebras of solutions to partial differential equations $\ldots \ldots \ldots \ldots \ldots \ldots \ldots \ldots \ldots \ldots \ldots \ldots \ldots$

Junichi Aramaki, On an extension of the Ikehara Tauberian theorem . . . . . 13

Giacomo Monti Bragadin, Abstract Riemannian stratifications .......... 31

Lawrence James Brenton and Richard Hill, On the Diophantine equation

$1=\sum 1 / n_{i}+1 / \prod n_{i}$ and a class of homologically trivial complex

surface singularities .................................41

C. Bruce Hughes, Controlled homotopy topological structures $\ldots . \ldots \ldots \ldots 69$

Peter Wilcox Jones and Takafumi Murai, Positive analytic capacity but zero Buffon needle probability . ...........................999

Gary M. Lieberman, Hölder continuity of the gradient at a corner for the

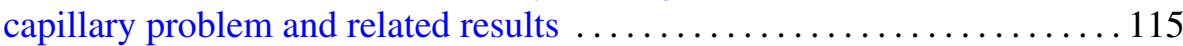

Feng Luo, Representing homology classes of $C \mathbf{P}^{2} \overline{C \mathbf{P}^{2}}$

Claudio Nebbia, Groups of isometries of a tree and the Kunze-Stein

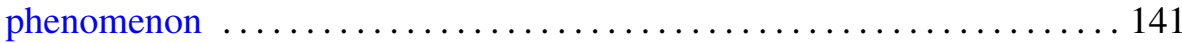

Stefan Richter, Unitary equivalence of invariant subspaces of Bergman and

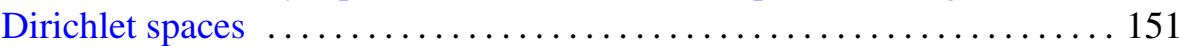

Paul Frederick Ringseth, The Selberg trace formula for groups without Eisenstein series ..................................... 157

Abderrazzak Sersouri, The Mazur property for compact sets $\ldots \ldots \ldots \ldots 185$ Alladi Sitaram, On an analogue of the Wiener Tauberian theorem for symmetric spaces of the noncompact type 